\title{
Hemorragia digestiva alta de origen no variceal: ¿Inyección, radiofrecuencia, polvos hemostáticos, métodos mecánicos?
}

\author{
Gretel B. Casillas-Guzmán*
}

Servicio de Endoscopia, Hospital General Regional No. 1 "Carlos MacGregor Sánchez Navarro”, Instituto Mexicano del Seguro Social, Ciudad de México, México

\section{Resumen}

La hemorragia digestiva alta no variceal (HDANV) sigue siendo una de las principales causas de tratamiento endoscópico, con una mortalidad entre 2-10\%. Además de los tratamientos endoscópicos convencionales, la utilización de polvos hemostáticos (Hemospray ${ }^{\circledR}$ ) ha mostrado seguridad y eficacia, principalmente en lesiones difíciles de tratar por métodos convencionales, su fácil aplicación y resultados lo ponen como una herramienta útil en el tratamiento de HDANV. Los clip OVESCO han demostrado ser útiles, eficaces y costo-efectivos como tratamiento de primera línea en el manejo de lesiones sangrantes, predominantemente las de alto riesgo de resangrado.

Palabras clave: Hemorragia digestiva alta. OVESCO. Úlcera péptica. Hemospray. Polvos hemostáticos.

\section{Introducción}

La HDANV afecta aproximadamente a 60-80/100,000 habitantes en Estados Unidos, y aunque se ha reducido su presentación con respecto a años previos, sigue siendo una causa frecuente de hospitalización, predominantemente en grupos de alto riesgo, causando la muerte en $2-10 \%$.

El manejo inicial se ha mantenido sin cambios en los últimos años, recomendándose en todos los casos mantener estabilidad hemodinámica, protección de la vía aérea (predominantemente en caso de hematemesis), resucitación con cristaloides y terapia de hemotransfusión restrictiva que se ha asociado a menor mortalidad y riesgo de resangrado ${ }^{2,3}$. En aquellos casos donde se requiere un manejo endoscópico, se cuentan con diversos métodos hemostáticos: inyección de adrenalina, métodos mecánicos y métodos térmicos, recientemente se ha extendido el uso de los polvos hemostáticos (Hemospray ${ }^{\circledR}$ ), por lo que revisaremos los trabajos relacionados con los métodos hemostáticos en hemorragia digestiva alta de origen no variceal.

\section{Polvos hemostáticos (Hemospray ${ }^{\circledR}$ )}

El Hemospray ${ }^{\circledR}$ o TC-325 es un polvo mineral con propiedades adsortivas diseñado para la hemostasia. El primer reporte del uso de polvos hemostáticos en humanos para tratar hemorragia péptica se publicó en $2011^{4}$. Desde entonces se ha probado su seguridad y eficacia. Las ventajas del Hemospray ${ }^{\circledR}$ son: Fácil aplicación que no requiere mucha experiencia, seguridad (no asociado a riesgo de embolización, oclusión o complicaciones pulmonares); cuando se ha realizado endoscopia al día siguiente de la aplicación, los polvos hemostáticos ya han desaparecido y dejan un campo

\section{Correspondencia:}


de visión limpio que permite adecuada visualización de la lesión para un tratamiento definitivo (en caso de requerirse). La desventaja: poca visibilidad al momento de la aplicación.

Bacarat, et al. realizaron un ensayo clínico donde compararon el uso de Hemospray ${ }^{\circledR}+$ inyección de adrenalina vs hemoclips + inyección de adrenalina para el manejo de pacientes con HDANV. Se incluyeron 39 pacientes con HDANV. Se aleatorizaron por método de computo. Todos los pacientes fueron sometidos a una endoscopia de seguimiento (second look). La etiología péptica fue la más frecuente. Se logró hemostasia primaria en todos los pacientes del grupo de Hemospray ${ }^{\circledR}$ y $90 \%$ del grupo de Hemoclip $(p=0.487)$. Cinco pacientes en el grupo de Hemospay ${ }^{\circledR}$ requirieron un segundo tratamiento hemostático en la endoscopia de seguimiento, y ninguno en el grupo de Hemoclip $(p=0.04)$. Las tasas de resangrado, cirugía de urgencia y mortalidad fueron similares en ambos grupos. No se presentó toxicidad o eventos adversos asociados al Hemospray ${ }^{\circledR}$. Los autores concluyen que el uso de Hemospray ${ }^{\circledR}$ presenta resultados similares cuando se compara con terapia convencional dual y tiene su tasa de hemostasia la convierte en una herramienta útil en situaciones de hemorragia severa 0 dificultad para localizar o tratar el sitio de hemorragia.

\section{Hemospray en endoscopia de urgencia}

M. Hussen ${ }^{6}$ en su trabajo presentado en la DDW 2019 reporta los resultados del uso de Hemospray en HDANV de origen péptico en un análisis del registro prospectivo multicéntrico internacional donde intervinieron 14 centros. Se incluyeron 202 pacientes, 7\% con uso concomitante de AINE. En promedio al ingreso con puntuación de Rockall de 7 (resangrado estimado en $25-40 \%$ ). La hemostasia inmediata a la aplicación de hemospray se logró en 178/202 (88\%), con tasa de resangrado de $17 \%$, mortalidad a los 7 días de $12 \%$ y a los 30 días de $22 \%$ (estimado por Rockall de 20$30 \%$ ). De acuerdo a la clasificación de Forrest, la hemostasia se logró en $34 / 39(87 \%)$ Forrest la, 99/117 (85\%) Forrest lb, 25/25 (100\%) Forrest Ila, $20 / 21$ (95\%) Forrest Ilb, con las siguientes tasas de resangrado: $24 \%, 15 \%, 9 \%$ y $22 \%$ en Forrest la, lb, Ila y Ilb respectivamente. De acuerdo a estos resultados, los autores concluyen que el Hemospray ${ }^{\circledR}$ logra tasas de hemostasia inmediata en úlceras pépticas en $88 \%$. En el análisis de subgrupos se observó que los pacientes que respondieron mejor fueron aquellos con terapia combinada (Hemospray ${ }^{\circledR}+$ adrenalina), úlceras esofágicas y úlceras Forrest lla.

\section{Clip ovesco}

Ermerak y colaboradores ${ }^{7}$ realizaron un estudio retrospectivo de casos y controles donde compararon la utilización de clip OVESCO vs tratamiento endoscópico convencional como tratamiento de primera lineal en hemorragia por úlcera péptica Se estudiaron 32 pacientes, se incluyeron 16 casos de HDA por úlcera péptica con aplicación de OVESCO como tratamiento de primera línea, reclutados en un periodo de 2 años, se parearon con un grupo control que recibió tratamiento convencional. Las indicaciones del uso de OVESCO fueron: vaso $>4 \mathrm{~mm}(\mathrm{n}=12)$, uso de anticoagulantes 0 antiplaquetarios $(n=3)$, sospecha de perforación $(n=2)$, acceso difícil a sitio de hemorragia $(n=1)$, o falla a otros tratamientos hemostáticos previos $(n=1)$. Se evaluó la tasa de resangrado a los 30 días, las readmisiones y la mortalidad a los 30 días. Los pacientes tratados con OVESCO tuvieron más probabilidad de ser pacientes hospitalizados (12 vs $4, p=0.005$ ), hipotensos (PAM 100 vs 118, $\mathrm{p}=0.04$ ), taquicárdicos (101 vs $95 \mathrm{lpm}, \mathrm{p}=0.02$ ) y tuvieron mayor proporción de lesiones Forrest I (12 vs 4). Se presentó resangrado dentro de los primeros 30 días en 1 paciente del grupo OVESCO vs 5 de la terapia convencional $(p=0.07)$. El caso de resangrado en el grupo OVESCO requirió angioembolización. Todos los casos de resangrado en el grupo control fueron manejados endoscópicamente. Las readmisiones durante los primeros 30 días, angioembolización o mortalidad no tuvieron diferencias estadísticamente significativas entre los grupos. Los autores concluyen que la aplicación de clip OVESCO tiende a reducir la tasa de resangrado, mientras que otras variables son similares a la terapia convencional, por lo que puede ser una ventaja en el tratamiento inicial de lesiones ulcerosas.

Por otro lado, Xinyao, et al. ${ }^{8}$ realizaron un estudio de costo-efectividad del uso de clip OVESCO comparado con hemoclips convencionales en caso de hemorragia ulcerosa péptica. Para el estudio utilizaron un modelo de toma de decisiones. Encontraron que el costo de un paciente con hemorragia recurrente fue de $\$ 8368.56$ USD utilizando clip OVESCO y $\$ 10528.55$ USD utilizando hemoclips convencionales. Los autores concluyen que la utilización de OVESCO se asocia a alta eficacia y bajo costo. 


\section{Conflicto de intereses}

No existen conflictos de interés. Sin patrocinio de la industria.

\section{Bibliografía}

1. Laine L, Yang H, Chang SC, Datto $C$. Trends for incidence of hospitalization and death due to $\mathrm{Gl}$ complications in the United States from 2001 to 2009. Am J Gastroenterol 2012; 107(8):1190-1195.

2. Sung JJ Y, et al. Asia-Pacific working group consensus on non-variceal upper gastrointestinal bleeding: an update 2018. Gut 2018; 67:1757-1768.

3. Odutayo A. Desborough M, et. al. Restrictive versus liberal blood transfusion for gastrointestinal bleeding: a systematic review and meta-analy- sis of randomised controlled trials. Lancet Gastroenterol Hepatol 2017; 2:354-60.

4. Sung JJ, Luo D, Wu JC, et al. Early clinical experience of the safety and effectiveness of Hemospray in achieving hemostasis in patients with acute peptic ulcer bleeding. Endoscopy 2011;43:291-5.

5. Baracat FI, de Moura DTH, et. al. Randomized controlled trial of hemostatic poder versus endoscopic clipping por non- variceal upper gastrointestinal bleeding. Surg Endosc 2019 Mar 1-8.

6. Hussein M. Alzoubaidi D. et al. Outcomes on the use of hemospray in upper gastrointestinal bleeds secondary to peptic ulcers: Prospective multicentre internacional hemospray registry S 2115 DDW2019.

7. Ermerak GG, Behary J, et al. Over the scope clips for primary therapy of bleeding upper gastrointestinal ulcers: A retrospective case control study. Mo1319 DDW 2019

8. Xinyao J, Kaktenbach T, et al. Over the scope clips for recurrente peptic ulcer bleeding is cost effective as compared to the through the scope clips. S 340 DDW2019. 